\title{
A Novel Approach for Modelling Complex Maintenance Systems Using Discrete Event Simulation
}

\author{
Abdullah Alrabghi* and Ashutosh Tiwari ${ }^{* *}$ \\ * Department of Industrial Engineering, University of Jeddah, Jeddah, Saudi Arabia \\ ** Manufacturing Department, Cranfield University, Cranfield, United Kingdom \\ aalrabghi@uj.edu.sa, a.tiwari@cranfield.ac.uk
}

\begin{abstract}
Existing approaches for modelling maintenance rely on oversimplified assumptions which prevent them from reflecting the complexity found in industrial systems. In this paper, we propose a novel approach that enables the modelling of non-identical multi-unit systems without restrictive assumptions on the number of units or their maintenance characteristics. Modelling complex interactions between maintenance strategies and their effects on assets in the system is achieved by accessing event queues in Discrete Event Simulation (DES). The approach utilises the wide success DES has achieved in manufacturing by allowing integration with models that are closely related to maintenance such as production and spare parts systems. Additional advantages of using DES include rapid modelling and visual interactive simulation. The proposed approach is demonstrated in a simulation based optimisation study of a published case. The current research is one of the first to optimise maintenance strategies simultaneously with their parameters while considering production dynamics and spare parts management. The findings of this research provide insights for non-conflicting objectives in maintenance systems. In addition, the proposed approach can be used to facilitate the simulation and optimisation of industrial maintenance systems.
\end{abstract}

Keywords: simulation, maintenance, Discrete Event Simulation, rapid modelling

CBM: Condition Based Maintenance

$\mathrm{CM}$ : Corrective Maintenance 
DES: Discrete Event Simulation

METBF: Mean Elapsed Time Between failures

OM: Opportunistic Maintenance

PM: Preventive Maintenance

TTF: Time To Failure

SA: Simulated Annealing

\section{Introduction}

Maintenance aims to retain assets in their operational states. It has emerged as a fundamental success ingredient in the modern industry. Enhancing the performance of maintenance systems through modelling and optimisation has been the focus of a large volume of published studies.

Analytical modelling of maintenance prevailed for a long time. The foundations were laid by researchers such as Barlow and Proschan [1]. This was later developed extensively to include a large number of maintenance optimisation models [2]. In general, most of these models are developed for a specific system compromising of a single unit or several identical components [3]. However, maintenance systems in the industry are becoming much more complex which limits the applicability of analytical modelling techniques $[4 ; 5]$.

The use of simulation to model maintenance systems is on the rise [6]. Simulation enables the modelling of complex behaviour and requires fewer assumptions compared to analytical modelling [7]. Although simulation is wellestablished in manufacturing in general, it appears to be still developing for maintenance [8].

Few researchers presented conceptual frameworks for modelling maintenance using simulation [9; 10]. These frameworks were developed for specific systems without detailing the modelling approach or providing numerical examples. 
Figure 1-1 shows a popular approach used in several DES studies [11-13]. The maintenance strategy and its parameters are entered manually in the simulation model. The simulation then samples a Time To Failure (TTF). If the scheduled maintenance intervention will occur before the failure, maintenance will be conducted resulting in updating the cost function, scheduling the next maintenance intervention and sampling a new TTF. However, if the breakdown occurred before the maintenance intervention, a CM will be conducted. The process continues running for the simulation run length. However, such approaches have a number of limitations. The maintenance system is modelled separately from other inter-related systems such as production and spare parts logistics. This in turn limits the utilisation of the dynamic feature of DES since interactions between machines and the effect of maintenance on production are not modelled. In addition, these approaches are used to model one maintenance strategy only. As a result, the choice of maintenance strategies cannot be optimised using frameworks such as the one suggested by Alrabghi and Tiwari [14].

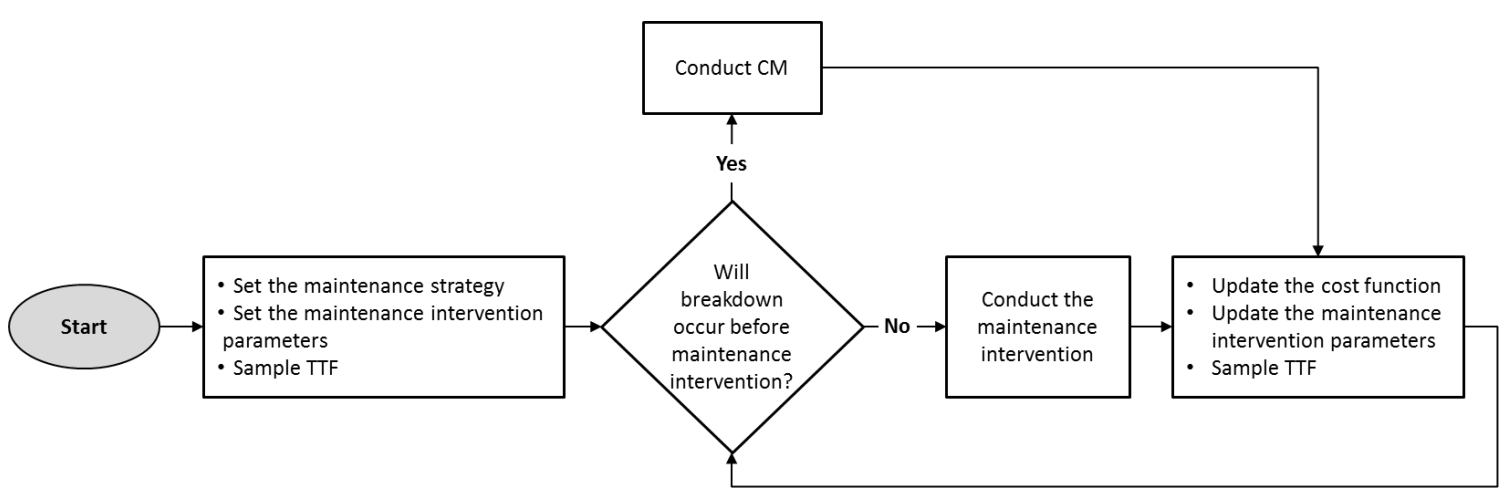

Figure 1-1 An existing modelling approach used in simulation studies. Adapted from [11-13]

Arab et al. [15] modelled both maintenance and production systems. However, they used manual DES calculations without utilising the strengths of available DES softwares such as rapid modelling and visual interactive simulation. On the other hand, Oyarbide-Zubillaga et al. [16] used an external tool to model the maintenance system and used that as an input to the DES model. 
The examination of surveys in the field $[4 ; 7 ; 17 ; 18]$ reveals a number of common research gaps relating to the modelling of maintenance systems:

1- Modelling the maintenance system in isolation of other significant and inter-related systems such as production and spare parts management.

2- Modelling various maintenance strategies and policies simultaneously.

3- Making over-simplifying assumptions resulting in a model that cannot be implemented in real-world systems. Such assumptions include perfect maintenance/ inspections, immediate maintenance actions and a singleunit system.

It appears as if these gaps are a result of the limitations present in the existing modelling approaches. Despite the potential of simulation to model complex maintenance systems, there remains a paucity of studies outlining adequate modelling approaches.

The present study fills a gap in the literature by proposing a modelling approach that can be used to model and optimise maintenance systems in practice. In addition to addressing the abovementioned limitations, the approach further exploits the advantages of DES such as rapid modelling and visual interactive simulation. As a result, the proposed approach is expected to pave the way for more advanced maintenance applications.

\section{Methodology}

\subsection{Modelling Maintenance Strategies}

The degradation of operational assets is inevitable. Maintenance actions are designed to improve the condition of assets to keep it in a functional state. Often maintenance strategies can be categorised into CM, PM and CBM. In $\mathrm{CM}$, the asset degrades until it breaks down unexpectedly. In some cases, the asset can breakdown suddenly without warnings. PM was introduced to minimise the effect of unscheduled breakdowns by interfering in a planned manner. CBM is an advanced strategy that aims to ensure maintenance intervention is conducted only when needed based on an analysis of the asset's condition. Predictive maintenance is seen as a part of CBM. The condition of 
assets is analysed to plan future maintenance actions. OM is closely related to both PM and CBM. Essentially, opportunities such as shutdowns are seized to preventively maintain an asset.

A considerable amount of literature has discussed the details of modelling each maintenance strategy and its implications on assets in the system. This includes the modelling of assets degradation, the degree to which a maintenance action can successfully detect a failure and the degree to which a maintenance action can restore the asset to as good as new [19;20].

However, in this paper we are considering a holistic view. Table 1 illustrates how the actions of a given maintenance strategy might affect assets in the system in different ways assuming the probability of occurrence of all failure modes does not change. The proposed approach enables the modelling of interactions amongst various maintenance strategies and their effects on the assets in the system. Thanks to the flexibility of DES, the proposed approach enables the construction of various maintenance systems based on models that appear in the literature. Classic examples include perfect/imperfect maintenance, perfect/imperfect inspections, dependencies amongst assets, effect of maintenance on product quality, effect of maintenance on production speed, various approaches to modelling asset degradation and inclusion/ exclusion of maintenance resources such as maintenance equipment, spare parts and technicians.

Table 1 Interactions amongst maintenance strategies

\begin{tabular}{|l|c|c|c|c|}
\hline $\begin{array}{l}\text { Might affect other maintenance } \\
\text { strategies on the same asset? }\end{array}$ & No & Yes & No & Yes \\
\hline $\begin{array}{l}\text { Might affect other maintenance } \\
\text { strategies on the other assets? }\end{array}$ & Yes & No & No & No \\
\hline
\end{tabular}

\subsection{Discrete Event Simulation}

The term DES refers to a modelling technique where only changes in system states are represented. Essentially, it creates a queue of events that affect the system state. These events are arranged based on their timings. The simulation then moves through these events and apply the changes on the system without 
modelling the time between any two events. Examples of such events in a typical manufacturing system include the arrival of a part, the start and finish of cycle times on machines and the occurrence of breakdowns. Therefore, it is a dynamic simulation technique where changes in the system are represented over time. The reader is referred to [21] for more details on DES.

DES has been applied successfully in a wide range of business and manufacturing applications. In fact, it is the most popular technique to model manufacturing systems [22]. The main features of typical DES software include modelling variability in statistical or empirical distributions and rapid modelling by providing built-in modules that accelerate the modelling process. In addition, a typical DES software enables visual interactive simulation where changes in the system are animated and users can interact during the simulation. Benefits of visual interactive simulation include better understanding of the model by visualising, interactive experimentation, improved communications to all stakeholders and the facilitation of model verification [23].

\section{A Novel Approach for Modelling Complex Maintenance Systems}

\section{Notations:}

MA: A single maintenance action resulting from a maintenance strategy.

SMA: A scheduled maintenance action resulting from a maintenance strategy.

$n$ : Total number of assets in the system.

$i$ : A single asset in the system where $i=1 \ldots n$

$T$ : simulation run length

A novel generic approach for modelling maintenance strategies is presented in Figure 3-1. The approach assumes the availability of a valid DES model for the manufacturing system in interest as well as the availability of required maintenance data. There are no restrictions on the number of assets in the manufacturing system or the number of maintenance strategies defined for each asset. The assets can be either identical or non-identical. Similarly, 
maintenance strategies can be the same for all machines or each asset can have its unique maintenance strategy.

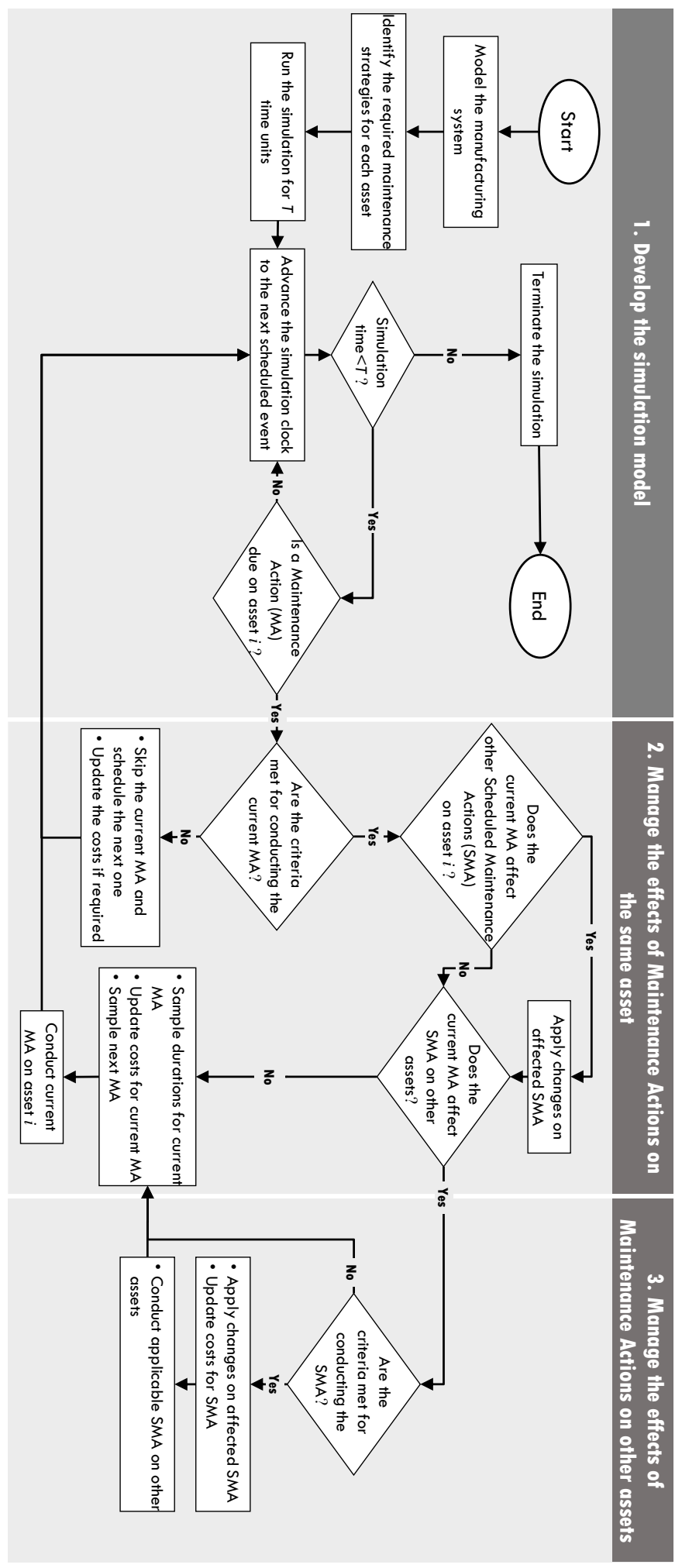

Figure 3-1 A generic approach for modelling maintenance strategies 
The approach consists of three steps as follows:

\section{Develop the simulation model}

The approach begins with modelling the manufacturing system. For example this might include assets, buffers and rules governing machine cycle times and movement of parts within the system.

The required maintenance strategies and policies are then identified for each asset. This includes defining parameters for statistical distributions required by each maintenance strategy to facilitate the modelling of variability in Maintenance Actions (MA) whenever they occur. For example, CM strategy requires the sampling from a statistical distribution to obtain Mean Elapsed Time Between Failures (METBF) each time the asset fails. In addition, a sampling from a statistical distribution is required to obtain the repair time. Other variables can be defined if required such as the cost of conducting each MA.

Other maintenance characteristics and assumptions can be modelled to reflect the required behaviour in the maintenance system. Examples include failure detection, effect of failures on production, administrative delays, safety rules and periodic tests. In addition, other aspects can be modelled as well such as spare parts, work shifts, repair teams and maintenance equipment.

When the simulation is run, the simulation clock is advanced to the next scheduled event. If a $M A$ is due on one of the assets in the system, the effects on the asset is managed in the next step.

\section{Manage the effects of Maintenance Actions on the same asset}

Whenever a $M A$ is due on asset $i$ in the system, a check is conducted to confirm that the criteria is met for the $M A$ to be executed. For instance, CBM requires the current relevant condition indicator to exceed a specific threshold in order for the MA to be conducted. Likewise, some PM policies will be skipped if the asset was broken down when the MA is due. Other criteria can be added depending on the maintenance system and its assumptions. Some examples include: availability of repair teams, availability of repair tools and availability of spare parts. If the criteria is not met, the current $M A$ will be skipped, costs will 
be updated if required and the next $M A$ of that maintenance strategy for asset $i$ will be scheduled.

However, if the criterion of conducting the $M A$ is met, a check will be conducted to determine if the current $M A$ was initiated by a maintenance strategy that affects other maintenance actions on the same asset. As illustrated in Table 1, maintenance strategies such as PM and CBM affect CM actions. The interactions between maintenance strategies can be implemented by accessing the event queue for asset $i$ and altering the timing of the relevant SMA. The effects of the current $M A$ on other assets in the system are managed in the next step.

The current $M A$ will be conducted on asset $i$ after scheduling the next $M A$. Whenever a $M A$ is conducted, costs are updated and samples are taken from the relevant distributions to schedule the new timing of an activity or define the repair time for a $M A$.

\section{Manage the effects of Maintenance Actions on other assets}

The current MA might affect SMA on other assets in the system. In that case, a check is conducted to confirm the criteria are met for the effects to take place. The event queue for these assets is accessed in order to apply the required changes. Steps 2 and 3 are repeated during the simulation every time a $M A$ is due on any asset in the system.

The next section presents detailed approaches for modelling common maintenance strategies namely Time-Based PM, OM and CBM with periodic inspections. These detailed approaches are special cases from the generic approach described in this section.

\subsection{Common Cases}

\subsubsection{Time-Based Preventive Maintenance}

In time-based PM, the asset is maintained periodically to minimise unexpected breakdowns. Figure 3-2 illustrates the approach for modelling a manufacturing system where time-based PM is applied. 


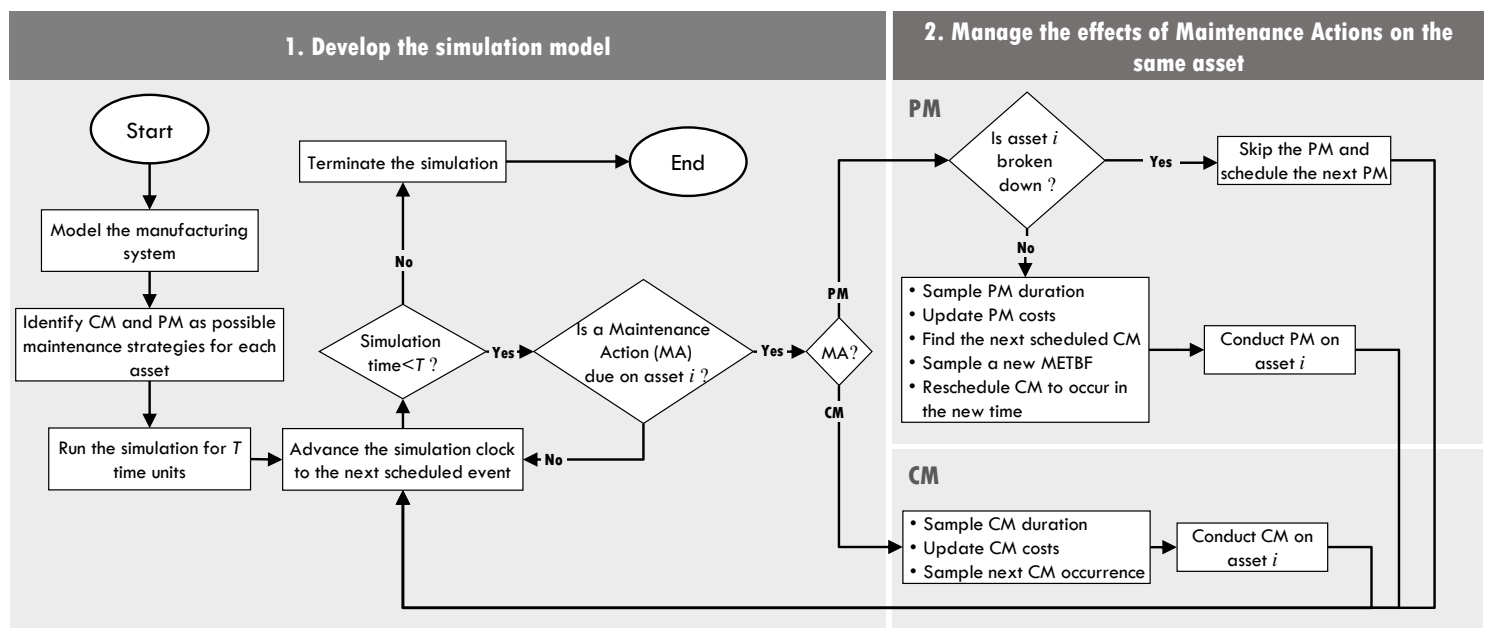

Figure 3-2 An approach for modelling time-based PM

\section{Develop the simulation model}

As assets can still breakdown unpredictably, both CM and PM are defined as possible maintenance strategies for each asset. Variables related to CM include METBF, repair times and CM costs whereas variables related to PM include PM frequency, repair times and PM costs. As the simulation clock advances, two maintenance strategies are possible on each asset, either CM or PM.

\section{Manage the effects of Maintenance Actions on the same asset}

When machines have an unscheduled breakdown, a CM duration is sampled to set the CM repair time, CM cost is added for asset $i$, and METBF is sampled to schedule the next CM. In addition, CM will be conducted on asset $i$ which means it will not be available for production.

However, when PM is due on asset $i$, PM duration is sampled to set the PM repair time and PM cost is added for asset $i$. Additionally, a sample from the METBF distribution will be drawn and the next CM breakdown will be changed to reflect the fact that PM has occurred. Finally, PM will be conducted on asset $i$ making it unavailable for use in the production system. Nonetheless, if the time of PM coincidentally occurred when asset $i$ is broken down, the current PM will be skipped and the next PM will run as scheduled. 
In this case, the third step of the approach is not required since none of the maintenance strategies considered for asset $i$ might have an effect on other assets in the system.

\subsubsection{Opportunistic Maintenance}

As a strategy, OM utilises the breakdown of as asset to maintain another asset. The approach for modelling OM is illustrated in Figure 3-3.

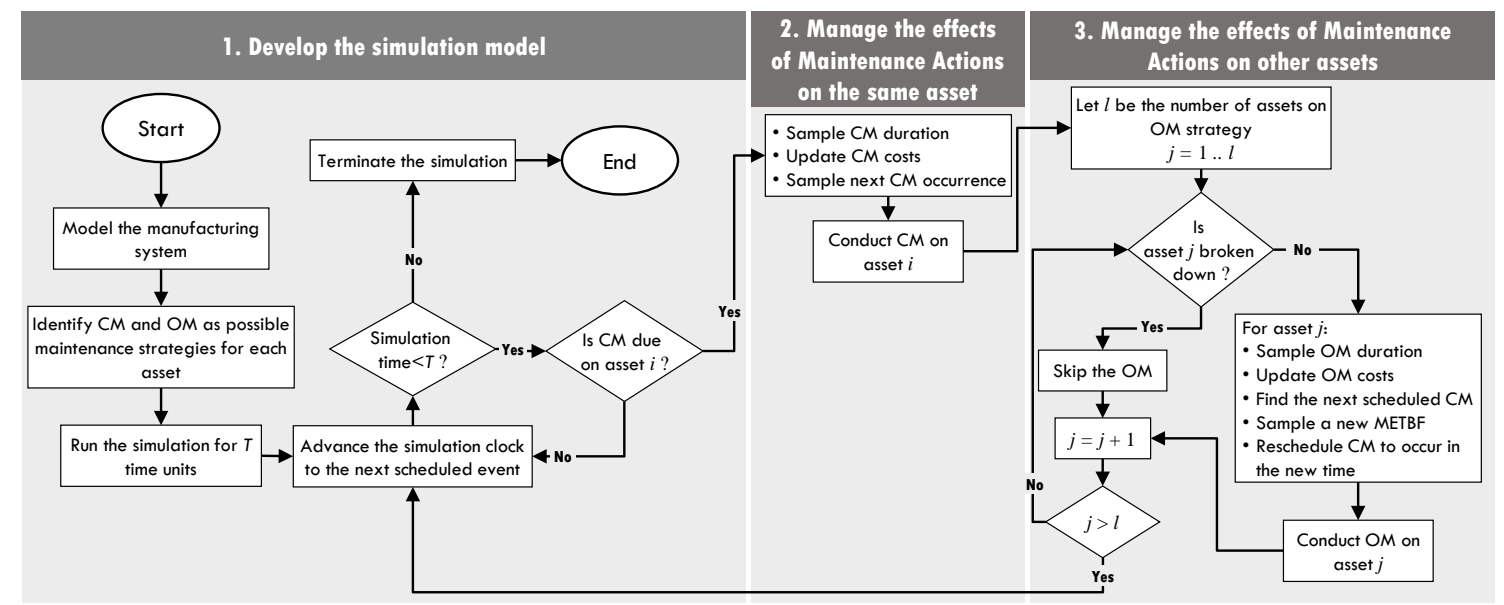

Figure 3-3 An approach for modelling OM

\section{Develop the simulation model}

$\mathrm{CM}$ and $\mathrm{OM}$ are identified as maintenance strategies for each asset. Variables related to CM include METBF, repair times and CM costs whereas variables related to $\mathrm{OM}$ include repair times and $\mathrm{OM}$ costs. When the simulation starts, the clock will advance running the simulation model until a $\mathrm{CM}$ becomes due to an asset in the system. The effects of CM on the same asset are managed in the next step.

\section{Manage the effects of Maintenance Actions on the same asset}

The asset subjected to $\mathrm{CM}$ will be made unavailable to conduct the required maintenance activities. Additionally, CM costs will be incurred and the next breakdown will be scheduled.

\section{Manage the effects of Maintenance Actions on other assets}


All other machines on OM strategy in the system will be stopped for OM during which an $\mathrm{OM}$ cost will be incurred and a sampling for OM duration will take place. In addition, the next breakdown will be rescheduled according to the METBF sampling. If OM coincidentally occurs while the asset has broken down it will be skipped without incurring any costs.

\subsubsection{Condition Based Maintenance with Periodic Inspections}

CBM strategy aims to further enhance the overall performance of assets by ensuring maintenance interventions are conducted only when needed. This is achieved by monitoring the condition of the asset and intervening when the condition exceeds a pre-set threshold. Figure 3-4 shows a modelling approach for CBM where the condition of assets is monitored by periodic inspections.

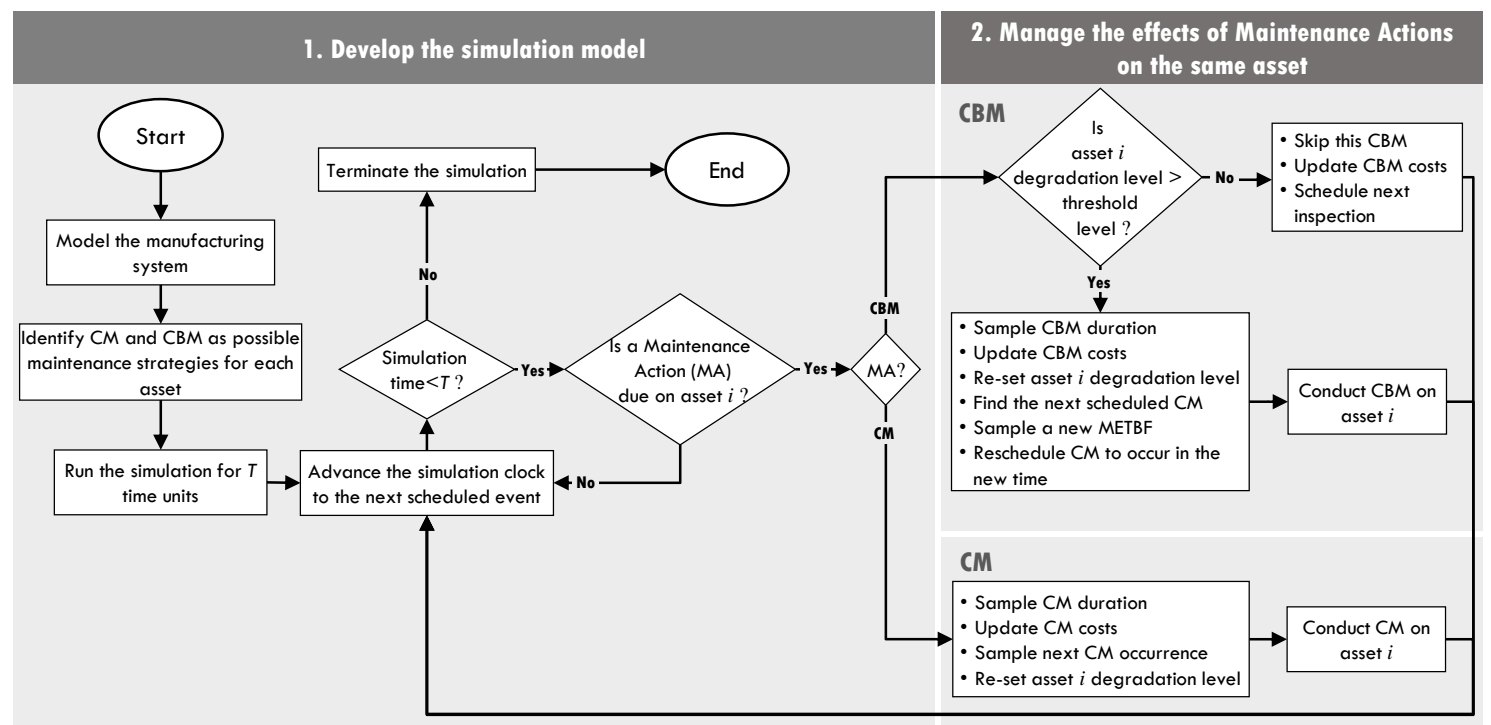

Figure 3-4 An approach for modelling CBM with periodic inspections

\section{Develop the simulation model}

Both $\mathrm{CM}$ and $\mathrm{CBM}$ are defined as maintenance strategies for each asset. CM variables include METBF, repair times and CM costs whereas CBM variables include inspection frequencies, inspection costs, CBM thresholds, CBM repair times and CBM costs. CM and CBM effects are managed in the next step.

\section{Manage the effects of Maintenance Actions on the same asset}


The path of $\mathrm{CM}$ is similar to the one discussed above in time-based PM. However, in this case, the degradation level for asset $i$ is set to the normal operation level after each $\mathrm{CM}$.

If the $M A$ was periodic inspection as part of the CBM strategy, a check is made to ensure the current wear level of asset $i$ exceeds the CBM threshold. A sampling from CBM duration will then take place to conduct CBM on asset $i$ in addition to updating CBM costs. The degradation level for asset $i$ is set to the normal operation level and the next $\mathrm{CM}$ will be rescheduled according to the sampling of METBF.

If an inspection reveals a value of degradation level less than the CBM threshold then CBM will be skipped and the next inspection will run as scheduled. However, CBM costs will be updated to add the incurred inspection cost.

In this case, the third step is not required as the considered strategies do not affect other assets.

\section{Case Study Application}

Notations:

$M S_{i} \quad$ Maintenance strategy for machine $i$

$P M f r e q i$ Preventive maintenance frequency for machine $i$

$Q_{i} \quad$ Order quantity for $S P_{i}$

$S_{i} \quad$ Reorder level for $S P_{i}$

$S P_{i} \quad$ Spare part for machine $i$

In this section, we demonstrate the application of the modelling approach through a simulation optimisation study of a published case [24]. In order to optimise the maintenance system, we follow the simulation-based optimisation framework suggested by Alrabghi and Tiwari [14]. 


\subsection{Simulation-Based Optimisation Framework for Maintenance Systems}

Alrabghi and Tiwari [14] suggested a framework for simulation based optimisation of maintenance systems (See Figure 4-1). It provides a systematic methodology that details the steps required to connect the simulation model to an optimisation engine. Not only it provides guidance in terms of formulating the optimal problem for the maintenance system at hand but it also provides support and assistance in defining the optimisation scope and investigating applicable maintenance strategies. Additionally, it considers current issues relating to maintenance systems both in research and in practise such as uncertainty, complexity and multi-objective optimisation.

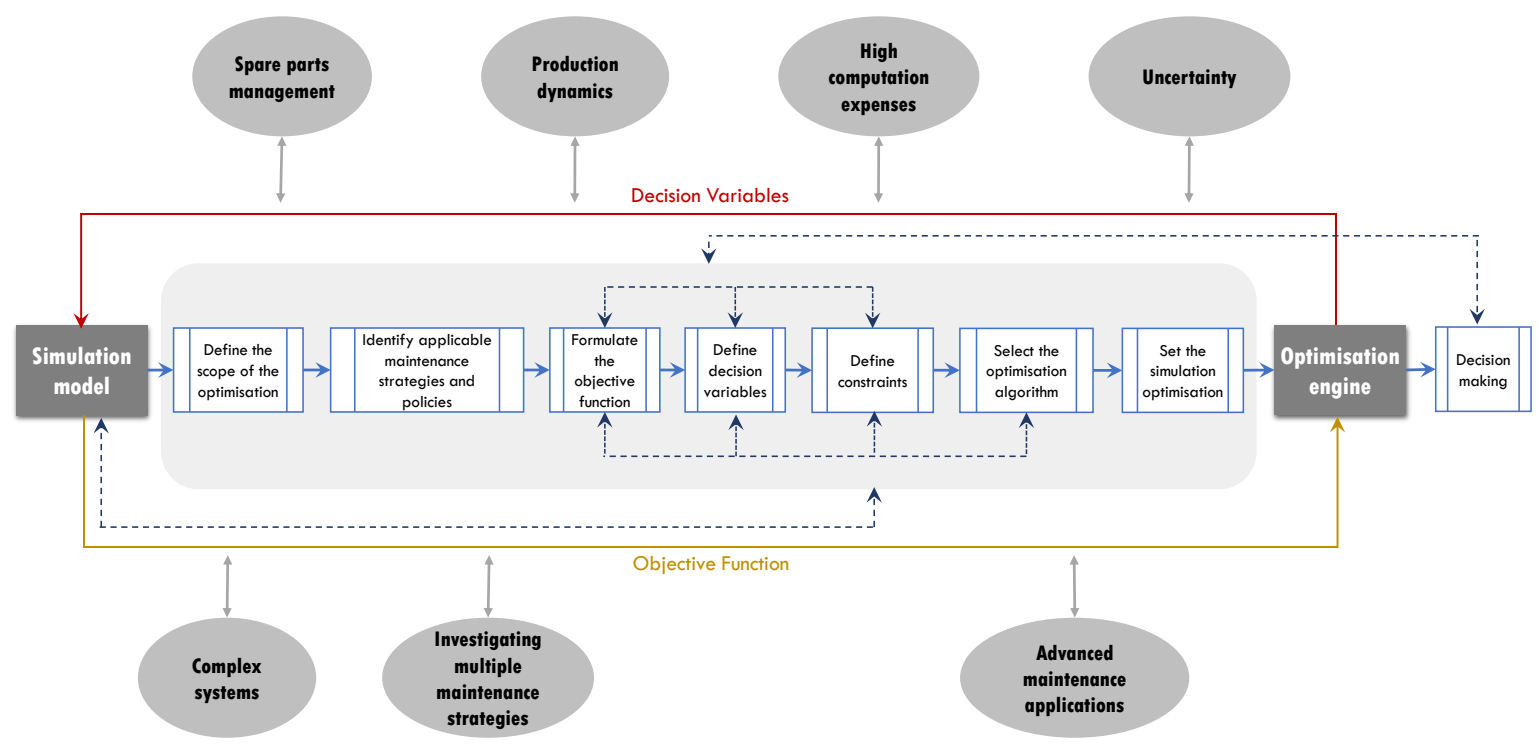

Figure 4-1 Simulation-Based Optimisation Framework for Complex Maintenance Systems on a High Level. Source: [14].

In addition to the first level shown in Figure 4-1, instructions for each step of the framework are provided in two more levels.

\subsection{Simulation Modelling}

Main assumptions include perfect maintenance where assets become as good as new following maintenance interventions and constant maintenance costs. As shown in Figure 4-2, Mean Elapsed Time Between Failures (METBF) is defined as the mean time between the start of any two consecutive failures. 

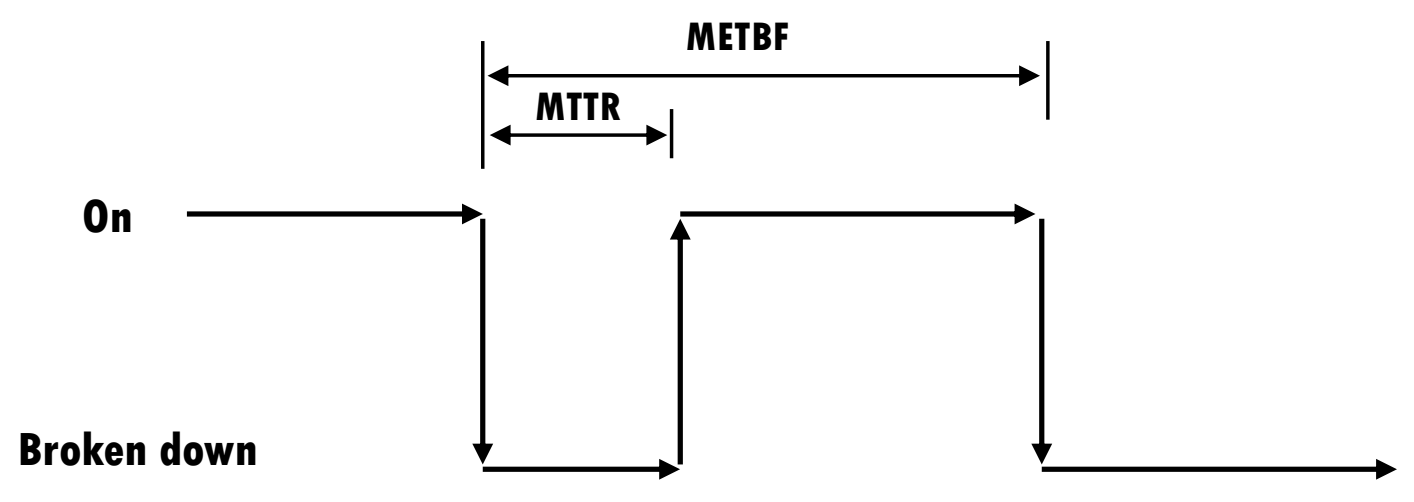

Figure 4-2 Mean Elapsed Time Between Failures (METBF) and Mean Time To Repair (MTTR) Notations

A Discrete Event Simulation model was developed using Witness 14 as it is already available within the research group. Each simulation is run for a number of replications to account for the variability arising from stochastic maintenance and production processes. A graphical method [23] is adopted to define a sufficient number of replications. It involves plotting the cumulative mean of the simulation output over a number of replications. The line becomes flat gradually which suggests that sufficient replications have been reached.

\subsection{Optimisation Technique}

Single objective optimisation was run using a Witness plug-in, Witness Optimizer which provides a number of optimisation algorithms including Simulated Annealing (SA), Hill Climb and Random Solutions. SA comes from the concept of the annealing process in metallurgy to harden metals. Metals are melted in high temperature at the start and then cooled gradually in a controlled environment to obtain desired attributes. It can be used to solve various types of problems including continuous, discrete and mixed-integer problems. Hill Climb is a local search heuristic algorithm that changes a single element in each iteration depending on the objective function performance. Random Solutions is simply randomising the values of decision variables without a structured algorithm to guide it to better solutions. This method can search globally but without the capability to learn from evaluations. 
Preliminary analysis was conducted by running the optimisation several times while changing the number of evaluation for each algorithm and monitoring the performance. It is observed that all three algorithms struggle to improve the objective function after around 150 evaluations. Therefore the maximum number of evaluations without improvements was set to 200 for all algorithms.

\subsection{Description of the Manufacturing System}

As illustrated in Figure 4-3, the manufacturing system consists of six nonidentical machines. There are buffers after each machine with the exception of machine 6 where processed parts are shipped out of the system. Spare provision policy is under continuous review and it includes $(s, Q)$ where $Q$ units are ordered each time the stock level reaches $s$. Lead times are stochastic and follow a uniform $(72,168)$ distribution. Only three technicians are available in the maintenance crew. Both Corrective Maintenance (CM) and Preventive Maintenance (PM) are applicable. The case study data was taken from [24].

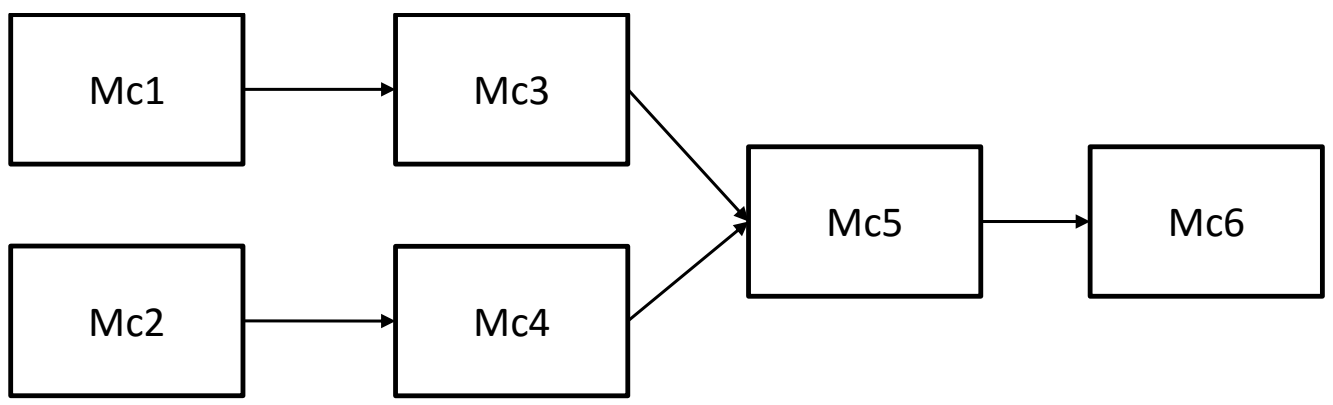

Figure 4-3 The Manufacturing System Layout. Source [24].

Cycle times follow Triangular distribution and vary between machines. Degradation patterns for machines are assumed to follow Weibull and Exponential distributions and vary between machines. Repair times for CM and PM tasks follow a Uniform distribution and vary between machines as well. All related distributions along with their parameters are shown in Table 2.

Table 2 Cycle times, breakdown patterns and repair times for the manufacturing system. Source [24].

\begin{tabular}{|l|l|l|l|l}
\hline Machine & Cycle time & Degradation & CM duration & PM duration \\
\hline
\end{tabular}




\begin{tabular}{|l|l|l|l|l|}
\hline & & pattern & & \\
\hline Mc1 & Triangle $(3,6,12)$ & Weibull(2, 3) & IUniform (1,3) & IUniform $(0.2,1)$ \\
\hline Mc2 & Triangle $(4,5,11)$ & Weibull(4, 2) & IUniform (1.2,3.5) & IUniform $(0.8,2.5)$ \\
\hline Mc3 & Triangle $(3,9,10)$ & Weibull(2, 2.5) & IUniform (1.7,2.3) & IUniform (1,1.5) \\
\hline Mc4 & Triangle $(5,9,10)$ & Weibull (3,1) & IUniform (1.5,3) & IUniform (1,1.5) \\
\hline Mc5 & Triangle $(7,9,13)$ & NegExp (2.5) & IUniform (0.7,2.5) & IUniform (0.5,1.6) \\
\hline Mc6 & Triangle (5,10,14) & NegExp (3) & IUniform (1,2.2) & IUniform (0.4,1.8) \\
\hline
\end{tabular}

The costs are constant during the simulation and are as follows:

- Corrective maintenance $=2000 /$ task

- Preventive maintenance $=750 /$ task

- Holding cost = 2/unit/hour

- $\quad$ order cost $=100 /$ order

- Unavailability penalty $=300$ / unavailable machine hour

\subsection{Results}

The simulation-based optimisation framework for maintenance systems is followed step by step as follows:

1. Define the scope of the optimisation: The assets in interest are assumed to be already identified. These are machines 1, 4 and 6. In this example, it is possible to alter the spare management policy. However, it is not possible to alter any production measures. Therefore the optimisation scope will include both maintenance and spare parts policy. Spare parts policy parameters for each machine, namely $s$ and $Q$ will be considered as decision variables.

2. Identify applicable maintenance strategies and policies: $\mathrm{CM}$ will be set as a possible maintenance strategy for all three machines. In addition, timebased PM is applicable in all three machines. Therefore, PM frequencies will be considered as decision variables. However, neither CBM nor selfmaintenance are applicable to any machine in this manufacturing system. 
The proposed approach described in section 3.1.1 is used to model the maintenance system.

3. Formulate the objective function: Production schedules are mostly stable and this optimisation does not aim to improve quality initiatives. Both minimising the cost and maximising the availability are considered important in this case. Machine unavailability incurs cost and can be incorporated in the cost function. Therefore, minimising the total cost will be the only objective. We consider the optimisation scope when detailing the cost function. As we are optimising maintenance and spare parts jointly, spare parts costs including the order and holding costs will be part of the cost function. In addition, both CM and PM maintenance costs will be detailed and added to the cost function. Hence, the objective function 'Total Cost' can be formulated as follows:

Minimise Total Cost= maintenance cost+ spare parts cost+ unavailability cost Where,

$$
\begin{gathered}
\text { Maintenance cost }=P M \operatorname{cost}+C M \text { cost, and, } \\
\text { Spare parts cost }=\text { order cost }+ \text { holding cost }
\end{gathered}
$$

4. Define the decision variables: Nine decision variables have been identified in the previous steps. These are the spare parts policy parameters $(s, Q)$ as well as the preventive maintenance frequency PMfreq for the selected machines $(I): 1,4$ and 6 . Three additional decision variables $\left(M S_{i}\right)$ are required to reflect the choice of maintenance strategy, either CM or PM. No more decision variables are required in this problem.

5. Define constraints: The maintenance system is well-known and therefore there is sufficient knowledge to define bounds for all decision variables. The reorder level $s_{i}$ can range between 0 to 15 while the order quantity $Q_{i}$ can range between 1 and 15. PM frequency for all machines $(P M$ freqi) can range between 1 and 3 weeks. MS will be either 0 if the selected maintenance strategy is $\mathrm{CM}$ and 1 if the selected maintenance strategy is PM. In addition, $M S$ will be incorporated in the variable bounds for PMfreq to ensure it results in 0 if the selected maintenance strategy is CM [25]. No other constraints are 
required at this problem. Therefore the problem can be formulated as follows:

Minimise Total Cost $=$ maintenance cost+ spare parts cost+ unavailability cost

$$
\begin{gathered}
1 \text { week }{ }^{*}\left(M S_{i}\right)<\text { PMfreq } i<3 \text { weeks }{ }^{*}\left(M S_{i}\right) \\
M S_{i}=0 \text { for } C M \text { or } 1 \text { for } P M \\
0<S_{i}<15 \\
1<Q_{i}<15 \\
\text { Where } i=1,4 \text { and } 6
\end{gathered}
$$

6. Select the optimisation algorithm: The current optimisation problem is single objective and requires a global search. Simulated Annealing (SA) suits the nature of the problem and it is available within the simulation software (WITNESS). The results of SA will be compared to two other optimisation algorithms available in WITNESS, namely Hill Climb and Random Solutions. Most of the algorithm settings are left to be set automatically including SA parameters such as splitting large variables, initial parameters, cooling rate and cooling steps, which control the rate at which the temperature is reduced. The maximum number of scenarios is set based on the number of possible solutions for the optimisation problem. As illustrated in Table 3 the solution space is vast which requires a large number of evaluations. Simplifying the problem may be possible which will be investigated in the next step. The maximum number of evaluations for all algorithms is set to 1,000 whereas up to 200 moves are allowed without improvement. 
Table 3 Possible solutions for the optimisation

\begin{tabular}{|c|c|c|c|c|c|}
\hline \multirow{2}{*}{$\begin{array}{c}\text { Variables } \\
\text { PMfreq }_{1}\end{array}$} & \multicolumn{2}{|c|}{ Ranges } & \multirow{2}{*}{$\begin{array}{c}\text { Current possible choices } \\
336 \\
\end{array}$} & \multirow{2}{*}{$\begin{array}{c}\text { possible choices } \\
\text { after } \\
\text { simplification } \\
14\end{array}$} & \multirow{2}{*}{$\begin{array}{c}\text { Remarks } \\
\text { changed from hour to day }\end{array}$} \\
\hline & 168 & 504 & & & \\
\hline $\mathrm{PMFreq}_{4}$ & 168 & 504 & 336 & 14 & changed from hour to day \\
\hline PMfreq $_{6}$ & 168 & 504 & 336 & 14 & changed from hour to day \\
\hline$s_{I}$ & 0 & 15 & 16 & 8 & changed from step 1 to step 2 \\
\hline$s_{4}$ & 0 & 15 & 16 & 8 & changed from step 1 to step 2 \\
\hline$s_{6}$ & 0 & 15 & 16 & 8 & changed from step 1 to step 2 \\
\hline$Q_{1}$ & 1 & 15 & 15 & 8 & changed from step 1 to step 2 \\
\hline$Q_{4}$ & 1 & 15 & 15 & 8 & changed from step 1 to step 2 \\
\hline$Q_{6}$ & 1 & 15 & 15 & 8 & changed from step 1 to step 2 \\
\hline$M_{1}$ & 0 & 1 & 2 & 2 & changed from step 1 to step 2 \\
\hline$M_{4}$ & 0 & 1 & 2 & 2 & no change \\
\hline$M_{6}$ & 0 & 1 & 2 & 2 & no change \\
\hline \multicolumn{3}{|c|}{ Possible solutions } & $4,195,092,529,152,000$ & $5,754,585,088$ & \\
\hline
\end{tabular}

7. Set the simulation optimisation: Variability analysis is conducted in order to set the required number of replications. As shown in Figure 4-4, the simulation is run repeatedly while the objective function (Total Cost) is recorded for each replication. In addition, a moving average is calculated. The moving average line seems to stabilise around the $16^{\text {th }}$ replication and hence the number of replications will be set to 16 to ensure we obtain a better estimate of (Total Cost) mean. Warm-up period is set to five days to avoid the initialisation bias since the manufacturing system starts with no parts in machines or buffers. The run length is set to one year to reflect the fact that the maintenance department plans annually for its operations. The cost baseline in the model before optimisation is $1,520,508$ cost units. 


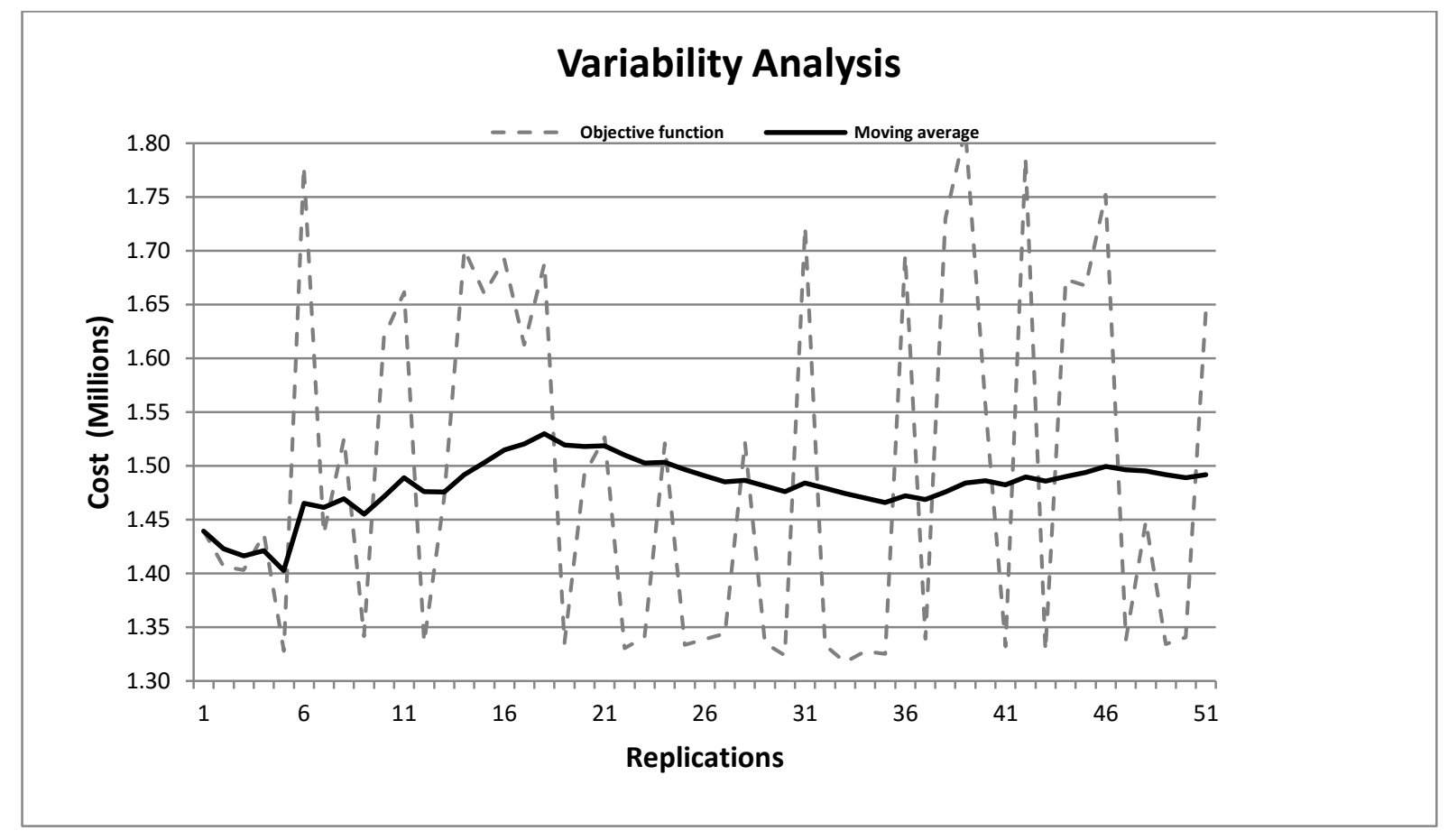

Figure 4-4 Variability analysis

One simulation run requires an average of $1: 17$ minutes on a PC with Intel Core i7-2600 CPU @ 3.40 GHz. At least several thousand evaluations are required for a problem with similar search space which consumes a long time. A thousand evaluations using SA are run with the current optimal formulation before attempting to simplify the problem. As shown in Figure 4-5, the optimisation resulted in cost reduction of $16.6 \%$ compared to the base model. The whole simulation optimisation required 18:45 hours to run. It is observed that small changes in the variables $P M f_{r e q} q_{\text {have insignificant effect on the total }}$ cost. Therefore it seems that simplifying the problem by discretising the decision variables will reduce the solution space with possibly minimal effect on the objective function. 


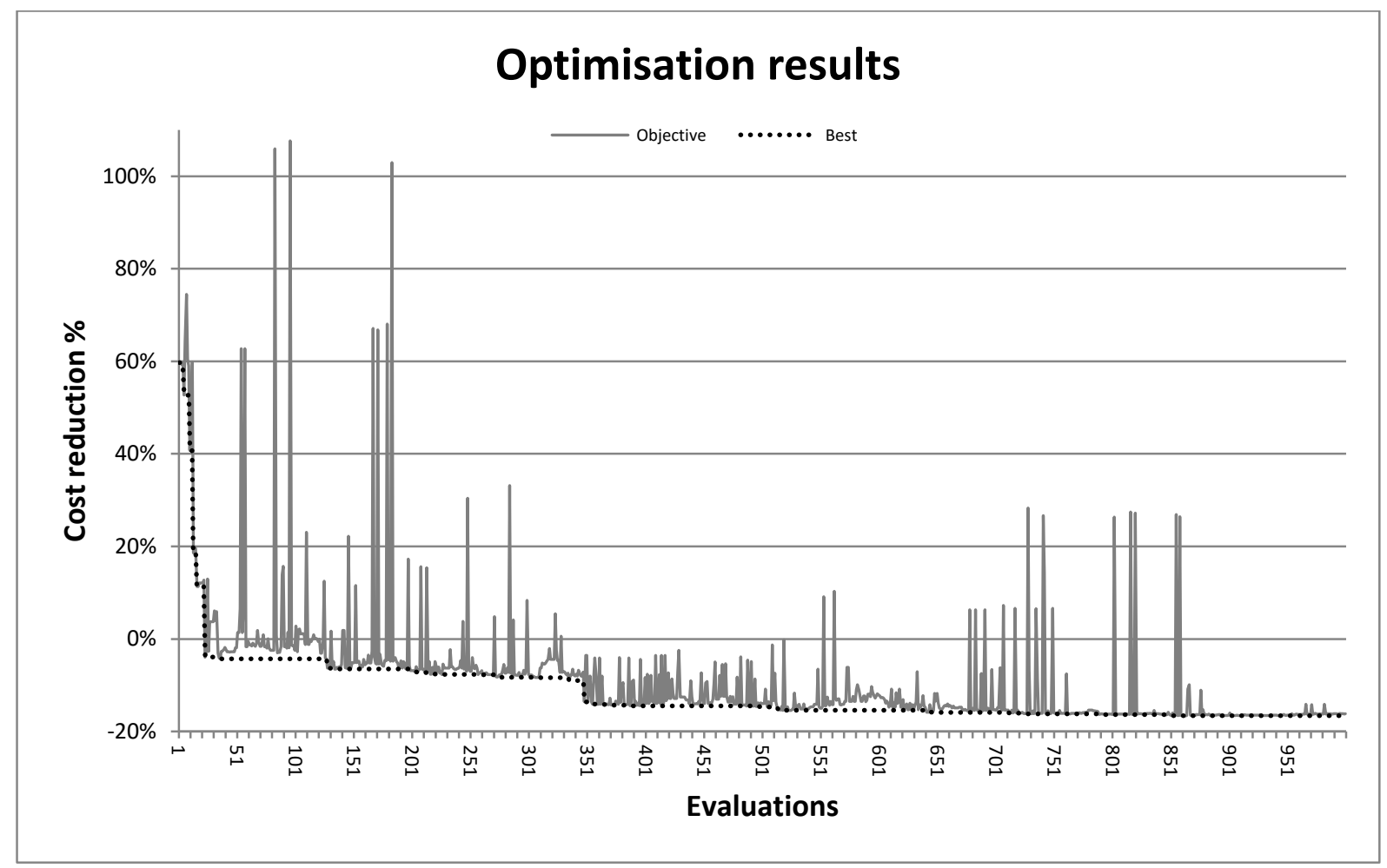

Figure 4-5 Optimisation results before simplifying the problem

The problem can be simplified by planning the PM for each machine by day instead of hour which reduces the possible values for each PMfreq from 336 to 14. However, the number of conflicts might increase where several events occurr at the same time within the simulation. In addition, both order quantity and order level can change two values at a time halving the number of their possible values. The solution space is reduced drastically as shown in Table 3. In addition to cost, the production throughput is considered an important measure to be taken into account when planning maintenance.

Table 4 presents a comparison of the best results achieved by each optimisation algorithm for the simplified problem along with computation time and number of evaluations. SA achieved the best result with $16.7 \%$ reduction in the total cost compared with the base model. The optimisation was terminated after 684 evaluations because it did not achieve an improvement in the objective function for 200 consecutive evaluations. The total computation time was 15 hours. It is interesting to note that by simplifying the problem, SA achieved a slightly better result consuming much less computation expenses. 
Table 4 Computation time and best results for different optimisation algorithms

\begin{tabular}{|l|l|r|c|c|}
\hline \multicolumn{2}{|c|}{ Optimisation algorithm } & \multicolumn{1}{|c|}{$\begin{array}{l}\text { Number of } \\
\text { evaluations }\end{array}$} & $\begin{array}{c}\text { Computation } \\
\text { time } \\
\text { (hh:mm) }\end{array}$ & $\begin{array}{c}\text { Best result } \\
\text { (cost reduction } \\
\%)\end{array}$ \\
\hline 1 & Random Solutions & 1,000 & $21: 56$ & $-14.5 \%$ \\
\hline 2 & Hill Climb & 459 & $09: 48$ & $-12.9 \%$ \\
\hline 3 & Simulated Annealing & 684 & $15: 00$ & $-16.7 \%$ \\
\hline
\end{tabular}

8. Decision making: Figure 4-6 compares the performance of the three optimisation algorithms. Hill climb converged rapidly but it struggled to achieve significant improvements after the 28th evaluations and it could not achieve any improvement after the 259th evaluation. This result may be explained by the fact that Hill Climb is not capable of conducting global search and therefore is bound to be trapped in a local minimum. This is further supported by the fact that both Random Solutions and SA were able to find better solutions.

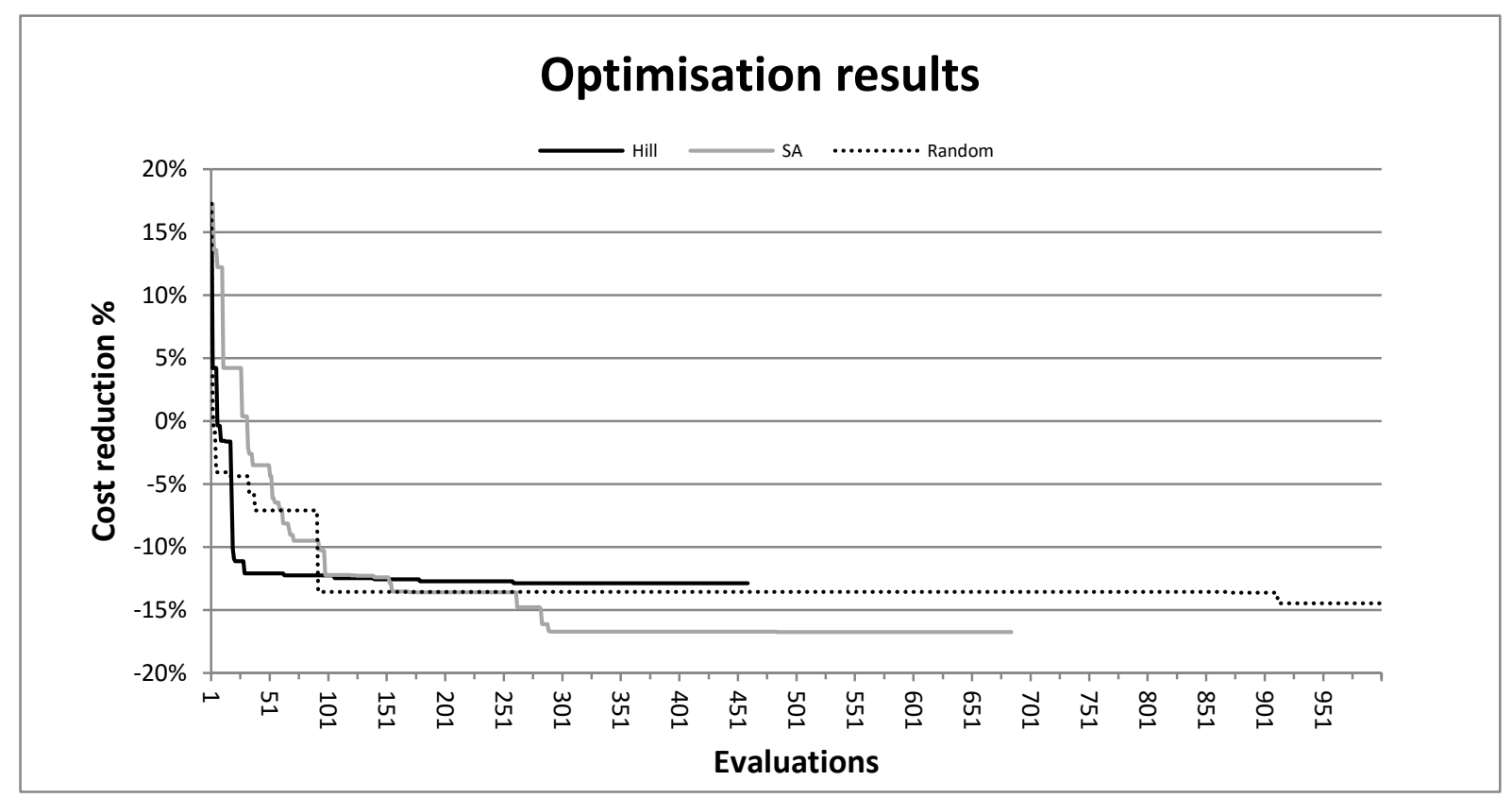

Figure 4-6 Comparison of the algorithms' performance

The firm's management might consider spending up to $10 \%$ more on maintenance if that will result in achieving higher productivity defined by the total throughput of the manufacturing system. Figure 4-7 below provides the outcomes obtained from plotting throughput vs. cost for the best $10 \%$ of the 
optimisation results. From the chart, it is apparent that the minimum cost corresponds with the maximum throughput. Therefore the firm's management would not have to attempt to balance throughput and maintenance cost for this problem.

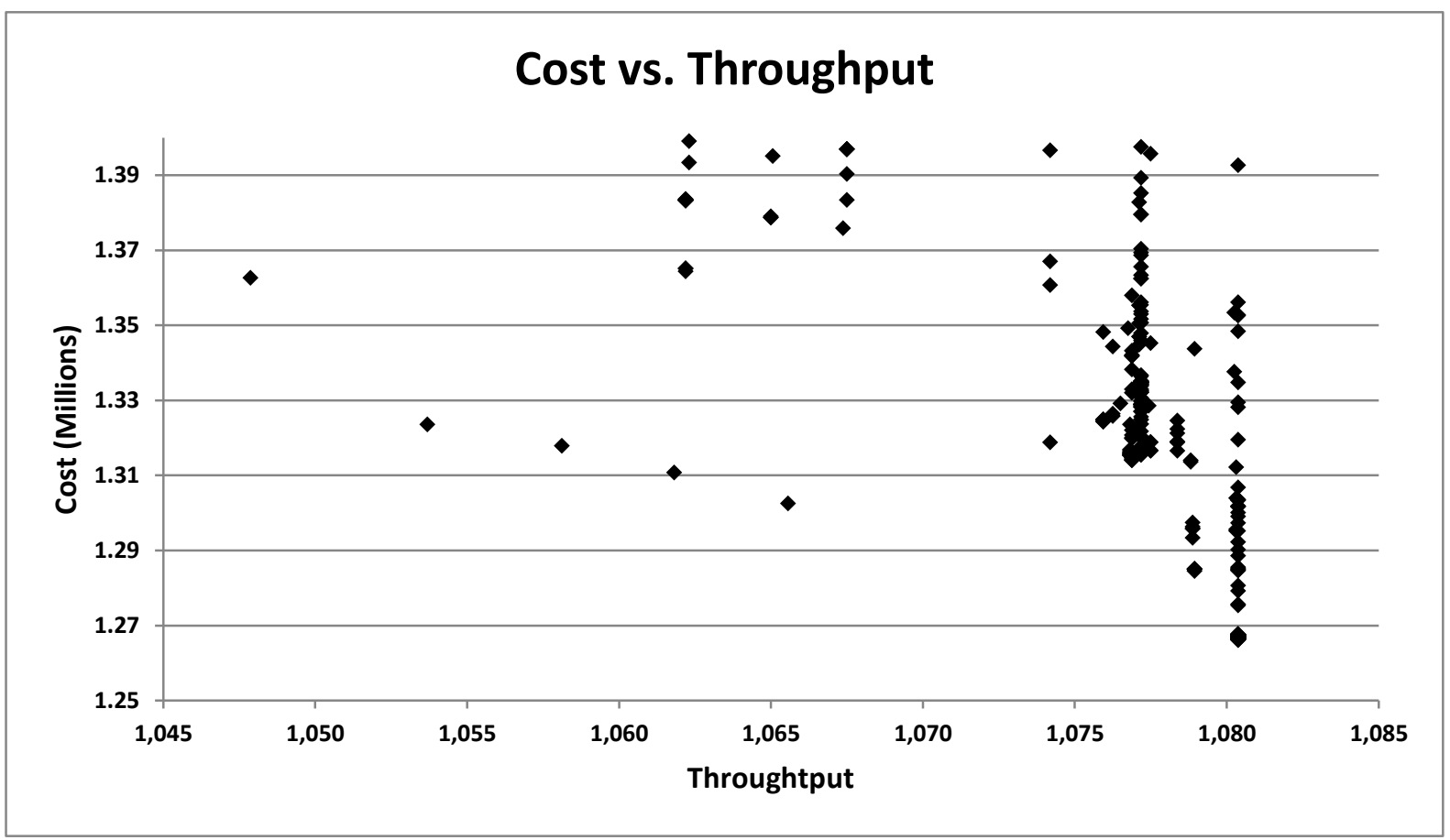

Figure 4-7 Plotting Cost vs. Throughput for the best $10 \%$ of the results

Nonetheless, the optimisation resulted in more than 100 solutions where the cost is in the range of $1 \%$ more than the minimum cost achieved while the throughput is 1080 which is the maximum value reached. Table 5 presents the top ten optimal solutions. From this data, we can see that the optimal maintenance strategy is PM for all machines. In addition, PM frequency does not change for the top ten solutions. Some spare management policy parameters such as $Q_{4}$ and $Q_{5}$ change resulting in a slight change in the cost function. Other considerations that were not taken into account in this study might affect the choice of the optimal solution such as quantity discounts. 
Table 5 Top ten optimal solutions

\begin{tabular}{|c|c|c|c|c|c|c|c|c|c|c|}
\hline Scenario & A & B & $\mathrm{C}$ & $\mathrm{D}$ & $\mathrm{E}$ & F & G & $\mathrm{H}$ & I & $\mathrm{J}$ \\
\hline Cost & \begin{tabular}{|l|}
$1,266,117$ \\
\end{tabular} & $1,266,142$ & $1,266,261$ & \begin{tabular}{|l|}
$1,266,273$ \\
\end{tabular} & $1,266,286$ & \begin{tabular}{|l|}
$1,266,292$ \\
\end{tabular} & \begin{tabular}{|l|}
$1,266,317$ \\
\end{tabular} & \begin{tabular}{|l|}
$1,266,404$ \\
\end{tabular} & $1,266,417$ & $1,266,417$ \\
\hline PMFreq1 & 384 & 384 & 384 & 384 & 384 & 384 & 384 & 384 & 384 & 384 \\
\hline PMFreq4 & 216 & 216 & 216 & 216 & 216 & 216 & 216 & 216 & 216 & 216 \\
\hline PMFreq6 & 216 & 216 & 216 & 216 & 216 & 216 & 216 & 216 & 216 & 216 \\
\hline $\mathbf{M S}_{1}$ & 1 & 1 & 1 & 1 & 1 & 1 & 1 & 1 & 1 & 1 \\
\hline $\mathbf{M S}_{4}$ & 1 & 1 & 1 & 1 & 1 & 1 & 1 & 1 & 1 & 1 \\
\hline $\mathbf{M S}_{6}$ & 1 & 1 & 1 & 1 & 1 & 1 & 1 & 1 & 1 & 1 \\
\hline $\mathbf{Q}_{1}$ & 5 & 5 & 5 & 5 & 5 & 5 & 5 & 5 & 5 & 5 \\
\hline $\mathbf{Q}_{4}$ & 5 & 5 & 3 & 5 & 3 & 5 & 3 & 11 & 11 & 11 \\
\hline $\mathbf{Q}_{6}$ & 11 & 7 & 11 & 7 & 7 & 15 & 11 & 5 & 11 & 11 \\
\hline $\mathbf{s}_{1}$ & 4 & 4 & 4 & 4 & 4 & 4 & 4 & 4 & 4 & 4 \\
\hline S4 & 2 & 2 & 2 & 2 & 2 & 2 & 4 & 2 & 4 & 2 \\
\hline S6 & 4 & 4 & 4 & 6 & 4 & 4 & 4 & 4 & 4 & 4 \\
\hline Throughput & 1080 & 1080 & 1080 & 1080 & 1080 & 1080 & 1080 & 1080 & 1080 & 1080 \\
\hline
\end{tabular}

\section{Discussion}

This study set out with the aim of developing an approach for modelling complex maintenance systems using DES. A generic approach as well as approaches for common maintenance strategies were presented.

The proposed approach enables the modelling of the complexity found in real maintenance systems. In particular, the approach enables the modelling of the following:

- Multi-unit manufacturing systems. Without restrictions on the number of units.

- Non-identical units. Without restrictions placed on the manufacturing or the maintenance characteristics of the units. In other words, each unit in the system can have its own stochastic manufacturing behaviour as well as its own stochastic maintenance behaviour.

- Several maintenance strategies and policies simultaneously. For the purpose of optimisation, each unit can have several applicable maintenance strategies. A variable can dictate the selection of a maintenance strategy. Therefore, the optimisation can result in a different strategy and different parameters for each unit in the system.

- Maintenance integrated with inter-related systems such as production and spare parts management. The proposed approach was designed for easy integration with already developed manufacturing systems. This 
enables the utilisation of the maturity stage DES has reached in production and logistics.

- Complex maintenance systems without over-simplified assumptions such as instantaneous repair, perfect maintenance or perfect inspection.

A typical DES software provides additional features that facilitate and speed up the modelling process. For example, machines, labour and breakdown modules are built in most of DES software packages. In addition, visual animation is displayed which enhance the communication between stakeholders and facilitate the validation process.

Accessing the event queue appeared to be the most suitable approach for the context of this approach. Other approaches were explored during the development of the proposed approach including forced breakdowns and using dummy machines to trigger machine actions. However, the alternative approaches resulted in much more complexity compared to the proposed approach.

The modelling approach was used in solving a simulation optimisation of maintenance in a published system. The current research is one of the first to optimise maintenance strategies simultaneously with their parameters while considering production dynamics and spare parts management. CM and PM were considered as possible maintenance strategies for selected assets in the manufacturing system. In addition, PM frequency was optimised in the same problem.

The findings of this research provide insights for non-conflicting objectives in maintenance systems. Minimising maintenance cost might in fact lead to maximum availability or maximum production throughput.

\section{Conclusions and Future Work}

Existing approaches for modelling maintenance rely on oversimplified assumptions which prevent them from reflecting the complexity found in industrial systems. Such assumptions are related to the scope of the simulation 
model, the number of assets, the manufacturing and maintenance characteristics of assets or the number of applicable maintenance strategies in the model.

In this paper, we develop a novel approach for modelling complex maintenance systems. The proposed approach enables the modelling of non-identical multiunit manufacturing systems without restrictions on either the maintenance or manufacturing characteristics. The approach can be integrated with DES manufacturing and spare parts models making it possible to build on the success DES achieved in these fields.

The case study application is one of the first to optimise maintenance strategies simultaneously with their parameters while considering production dynamics and spare parts management. The findings of this research provide insights for non-conflicting objectives in maintenance systems. This would be a fruitful area for further work.

This research will serve as a base for future maintenance optimisation studies. The ability of modelling simultaneous maintenance strategies makes it possible to conduct simulation-based optimisation studies where maintenance strategies are optimised for each asset in the system. In other words, the optimisation engine will explore various maintenance strategies along with its parameters for each asset.

Further research can be undertaken to implement the approach to industrial case studies. Additionally, more approaches can be developed for more common cases such as CBM with on-line monitoring and predictive maintenance. Furthermore, the modelling of aged-base models using the proposed approach needs to be investigated.

\section{Acknowledgements}

The authors would like to thank the University of Jeddah for funding this research. 


\section{REFERENCES}

[1] Barlow, R. E. and Proschan, F. (1965), Mathematical theory of reliability, Wiley, New York.

[2] Dekker, R. (1996), "Applications of maintenance optimization models: a review and analysis", Reliability Engineering and System Safety, vol. 52, no. 3, pp. 229-240.

[3] Van Horenbeek, A., Buré, J., Cattrysse, D., Pintelon, L. and Vansteenwegen, P. (2013), "Joint maintenance and inventory optimization systems: a review", International Journal of Production Economics, vol. 143, no. 2, pp. 499-508.

[4] Nowakowski, T. and Werbinka, S. (2009), "On problems of multicomponent system maintenance modelling", International Journal of Automation and Computing, vol. 6, no. 4, pp. 364-378.

[5] Nicolai, R. P. and Dekker, R. (2008), "Optimal maintenance of multicomponent systems: a review", in Kobbacy, K. and Murthy, D. N. (eds.) Complex system maintenance handbook, Springer, London, pp. 263-286.

[6] Sharma, A., Yadava, G. and Deshmukh, S. (2011), "A literature review and future perspectives on maintenance optimization", Journal of Quality in Maintenance Engineering, vol. 17, no. 1, pp. 5-25.

[7] Alrabghi, A. and Tiwari, A. (2015), "State of the art in simulation-based optimisation for maintenance systems", Computers and Industrial Engineering, vol. 82, pp. 167-182.

[8] Alabdulkarim, A. A., Ball, P. D. and Tiwari, A. (2014), "Influence of resources on maintenance operations with different asset monitoring levels: A simulation approach", Business Process Management Journal, vol. 20, no. 2, pp. 195-212.

[9] Warrington, L., Jones, J. A. and Davis, N. (2002), "Modelling of maintenance, within discrete event simulation", Annual Reliability and Maintainability Symposium, The International Symposium on Product Quality and Integrity, 28-31 January 2002, Seattle, WA, IEEE, Piscataway, NJ, pp. 260.

[10] Duffuaa, S., Ben-Daya, M., Al-Sultan, K. and Andijani, A. (2001), "A generic conceptual simulation model for maintenance systems", Journal of Quality in Maintenance Engineering, vol. 7, no. 3, pp. 207-219.

[11] Roux, O., Jamali, M. A., Kadi, D. A. and Châtelet, E. (2008), "Development of simulation and optimization platform to analyse 
maintenance policies performances for manufacturing systems", International Journal of Computer Integrated Manufacturing, vol. 21, no. 4, pp. 407-414.

[12] Van Horenbeek, A. and Pintelon, L. (2012), "Optimal prognostic maintenance planning for multi-component systems", European Safety and Reliability Conference: Advances in Safety, Reliability and Risk Management, ESREL 2011, 18-22 September 2011, Troyes, France, Taylor \& Francis Group, London, pp. 910-917.

[13] Asadzadeh, S. M. and Azadeh, A. (2014), "An integrated systemic model for optimization of condition-based maintenance with human error", Reliability Engineering and System Safety, vol. 124, pp. 117-131.

[14] Alrabghi, A. and Tiwari, A. (2016), "A novel framework for simulationbased optimisation of maintenance systems", International Journal of Simulation Modelling, vol. 15, no. 1, pp. 16-28.

[15] Arab, A., Ismail, N. and Lee, L. S. (2013), "Maintenance scheduling incorporating dynamics of production system and real-time information from workstations", Journal of Intelligent Manufacturing, vol. 24, no. 4, pp. 695695.

[16] Oyarbide-Zubillaga, A., Goti, A. and Sanchez, A. (2008), "Preventive maintenance optimisation of multi-equipment manufacturing systems by combining discrete event simulation and multi-objective evolutionary algorithms", Production Planning and Control, vol. 19, no. 4, pp. 342-355.

[17] Wang, H. (2002), "A survey of maintenance policies of deteriorating systems", European Journal of Operational Research, vol. 139, no. 3, pp. 469-489.

[18] Ding, S. and Kamaruddin, S. (2015), "Maintenance policy optimization: literature review and directions", The International Journal of Advanced Manufacturing Technology, vol. 76, no. 5-8, pp. 1263-1283.

[19] Van Horenbeek, A., Pintelon, L. and Muchiri, P. (2010), "Maintenance optimization models and criteria", International Journal of Systems Assurance Engineering and Management, vol. 1, no. 3, pp. 189-200.

[20] Wang, W. (2012), "An overview of the recent advances in delay-timebased maintenance modelling", Reliability Engineering and System Safety, vol. 106, pp. 165-178.

[21] Robinson, S. (2014), "Discrete event simulation: A primer", in Brailsford, S., Churilov, L. and Dangerfield, B. (eds.) Discrete-Event Simulation and System Dynamics for Management Decision Making, John Wiley \& Sons Ltd, Chichester, pp. 10-25. 
[22] Jahangirian, M., Eldabi, T., Naseer, A., Stergioulas, L. K. and Young, T. (2010), "Simulation in manufacturing and business: A review", European Journal of Operational Research, vol. 203, no. 1, pp. 1-13.

[23] Robinson, S. (2007), Simulation : the practice of model development and use, Wiley, Chichester.

[24] Alrabghi, A., Tiwari, A. and Alabdulkarim, A. (2013), "Simulation based optimization of joint maintenance and inventory for multi-components manufacturing systems", Proceedings of the 2013 Winter Simulation Conference, 8-12 December 2013, Washington DC, IEEE, Piscataway, NJ, pp. 1109-1119.

[25] Sarker, R. A. and Newton, C. S. (2008), Optimization modelling : a practical approach, CRC Press, Boca Raton. 
2016-06-08

\title{
A novel approach for modelling complex maintenance systems using discrete event simulation
}

\author{
Alrabghi, Abdullah Omar
}

Elsevier

Abdullah Alrabghi, Ashutosh Tiwari, A novel approach for modelling complex maintenance systems using discrete event simulation, Reliability Engineering \& System Safety, Volume 154, October 2016, Pages 160-170

http://dx.doi.org/10.1016/j.ress.2016.06.003.

Downloaded from Cranfield Library Services E-Repository 